\title{
Niñez y Ejército. Historia de los niños-cadetes del Colegio Militar Eloy Alfaro de Quito, Ecuador, 1969 - 1972
}

\author{
Childhood and Army. The Story of Army Cadet Children at the Eloy Alfaro \\ Military School in Quito, Ecuador, 1969 - 1972
} Crianças e Exército. História dos meninos-cadetes do Colégio Militar Eloy
Alfaro de Quito, Equador, 1969-1972

\section{Kléver Antonio Bravo ${ }^{1}$ \\ Carlos Gutiérrez Álvarez ${ }^{2}$ \\ Universidad de las Fuerzas Armadas-ESPE, Ecuador}

Recepción: 15/05/2017

Evaluación: 30/06/2018

Aceptación: 29/06/2018

Artículo de Investigación - Reflexión

DOI: $10.19053 / 01227238.8582$

\section{RESUMEN}

Pasadas cinco décadas, se recupera la de ilusiones, sacrificios, travesuras, malmemoria de cincuenta y cinco niños que, ingresados al Colegio Militar Eloy Alfaro, inauguraron, en 1969, la educación secundaria que solo funcionaba desde el cuarto curso en la Escuela Militar, específicamente para la formación de oficiales del Ejército ecuatoriano.

Dado que el grueso de este trabajo fue construido a partir de entrevistas, nos fue posible revelar las vivencias de tres años tratos y nuevos conocimientos de aquellos niños-cadetes que iniciaron el primer curso bajo el manto de un sentimiento de patriotismo y otras creencias cívicas que debían ser forjadas en ellos.

Esta investigación testimonial se llevó a cabo en la ciudad de Quito, teniendo como muestra a un grupo de seis oficiales del Ejército ecuatoriano que protagonizaron esta historia, cuyo financiamiento

1 Máster y Ph.D. en Historia de América Latina por la Universidad Pablo de Olavide, Sevilla-España; máster en Relaciones Internacionales por la Universidad San Francisco de Quito; diplomado superior en Comunicación Social por la Universidad Andina Simón Bolívar; docente titular principal en la Universidad de las Fuerzas Armadas; Miembro de número de la Academia Nacional de Historia del Ecuador; profesor de la Academia de Guerra del Ejército ecuatoriano; coordinador de investigaciones del Departamento de Seguridad y Defensa de la Universidad de las Fuerzas Armadas; autor de 15 libros y varios artículos científicos sobre Historia Militar ecuatoriana. Correo electrónico: kabravo@espe.edu.ec

2 Máster en Educación, docente de la Universidad de las Fuerzas Armadas-ESPE, Ecuador, línea de investigación, Historia de la Educación en el Ecuador. Correo electrónico: clgutiérrez@espe.edu.ec 
nunca constituyó un obstáculo por ser la entrevista una de las fuentes primarias.

Palabras clave: Revista de Historia de la Educación en Latino América; Niños-cadetes; Colegio Militar; vivencias; testimonios.

\section{ABSTRACT}

After five decades, the memory of fifty-five children is recovered. Once they entered the Eloy Alfaro Military School in 1969, they inaugurated the secondary education that was only offered from the fourth grade at the Military School, specifically intended for the training of Ecuadorian Army officers. Since a large extent of this work was built on interviews, we were able to reveal the experiences of three years of illusions, sacrifices, pranks, abuse, and new knowledge of those cadet children who started the first course under the mantle of a patriotism feeling, and other civic beliefs, that should be forged in them.

This testimonial research was carried out in the city of Quito. The sample consisted of a group of six officers of the Ecuadorian Army who starred in this story. The financing was never an obstacle because the interview was one of the primary sources.

Keywords: Journal History of Latin American Education; Army Cadet Children; Military school; Life experiences; testimonies.

\section{RESUMO}

Passadas cinco décadas, recupera-se a memória de cinquenta e cinco meninos que, ingressados ao Colégio Militar Eloy Alfaro, inauguraram, em 1969, a educação secundária que funcionava somente desde o quarto curso na Escola Militar, especificamente para a formação de oficiais do Exército equatoriano. Dado que a maior parte desse trabalho foi construída a partir de entrevistas, nos foi possível revelar as vivências de três anos de ilusões, sacrifícios, travessuras, maus tratos e novos conhecimentos daqueles meninos-cadetes que iniciaram o primeiro curso sob o manto de um sentimento de patriotismo e outras ciências cívicas que deviam ser forjadas neles. Um sentimento de patriotismo e outras crenças cívicas que deviam ser forjada neles. Esta pesquisa testemunhal foi realizada na cidade de Quito, tendo como amostra um grupo de seis oficiais do Exército equatoriano que protagonizaram esta história, cujo financiamento nunca constituiu um obstáculo pelo fato de a entrevista ser uma das fontes primárias.

Palavras-chave: Revista de História da Educação Latino-americana; Meninos-cadetes; cadetes; Colégio Militar; vivencias; testemunhos.

\section{INTRODUCCIÓN}

\section{Problema}

La historia militar en el Ecuador ha permanecido en la retaguardia y mucho más en lo que se refiere a estudios críticos y descriptivos de los institutos de formación militar pertenecientes al Ejército ecuatoriano. No existe investigación alguna en este campo y los centros de investigación histórica poco o nada se han intere- sado por estudiarlo y menos aún publicarlo y, por supuesto, han dejado pasar una faceta tan interesante como la del Colegio Militar Eloy Alfaro. A lo anterior se suma la escasa atención de los uniformados a la cultura de conservación de la documentación, lo cual se explica de mejor manera con la mala costumbre de que "papeles que no sirven, a la pira o a la basura". 
Dicho vacío histórico justifica este artículo y, muy en particular, la necesidad de conservar la narrativa de esos niños-cadetes que conformaron la primera promoción de bachilleres del Eloy Alfaro.

En este contexto se puede comprobar que no existe publicación alguna de este tema, a lo que se añade que sus mismos actores, tras cinco décadas, apenas si conservan el recuerdo de cuando fueron cadetes, expresión compuesta que puso de manifiesto el coronel Enrique Rodríguez, director del colegio militar en aquel año de 1969, cuando daba el discurso de bienvenida e inauguración del nuevo año lectivo ${ }^{3}$.

\section{Objetivo}

El presente trabajo de investigación histórica tiene como objetivo, a través de fuentes testimoniales y bibliográficas, preservar la memoria de los niños-cadetes que ingresaron al colegio militar en el año 1969, tomando en cuenta que ese grupo de cincuenta y cinco niños, con un promedio de edad de doce años, inauguraba el ciclo básico de educación secundaria en el colegio militar, determinando así el grado de percepción sobre la vida diaria, el grado de afectividad y en qué medida influenció en sus vidas el paso por este colegio durante aquellos tres primeros años.

\section{Metodología y fuentes}

En cuanto a la metodología, este trabajo tiene carácter descriptivo y narrativo, por lo que merece decir que se ha realizado con una metodología cualitativa de observación directa, teniendo como fuentes primarias y secundarias los archivos del Colegio Militar Eloy Alfaro, testimonios directos y algunas publicaciones militares de la época, por cierto, muy escasas. Por supuesto, la fuente más efectiva para obtener la información fue la entrevista individual a los protagonistas, señores oficiales del Ejército ecuatoriano en servicio pasivo que hoy tienen una edad promedio de sesenta años y que al momento de la entrevista afirmaron que, para ellos, "no ha habido cosa tan emocionante en los últimos tiempos" que recordar aquellos años. A este grupo se sumaron otras personas entrevistadas que fueron parte de ese proceso educativo y de formación militar, esto es, instructores y brigadieres de la época que dieron luces a este trabajo de investigación.

\section{Hallazgos}

No se podría pasar por alto a la Grecia clásica, específicamente a Esparta, la ciudad-estado de los guerreros. Se dice que allí, cuando nacía un niño, era llevado a un consejo de ancianos para ser examinado. Si el niño tenía algún defecto

3 Entrevista del 29 de julio de 2017 con el teniente coronel Jorge Romero, padre de familia. 
era arrojado a los barrancos del monte Taigeto, al sur de Grecia. Empero, si el niño nacía sano y robusto, regresaba al regazo de su madre hasta los siete años, tiempo en el cual iniciaba su formación militar con ejercicios físicos y manejo de armas. En definitiva, se formaba al niño para convertirlo en un valeroso guerre$\mathrm{ro}^{4}$.

Por otra parte, Juan Marchena, el gran historiador militar sevillano, nos recuerda que en los regimientos militares españoles de inicios del siglo xviII, ya existían plazas para cadetes de entre 20 y 25, cuyos padres eran los oficiales o miembros de la nobleza local o regional. Estos cadetes tenían su instructor, que era el encargado de su entrenamiento castrense, disciplina y conocimiento de los reglamentos. Desfilaban, marchaban e incluso combatían en las compañías en las cuales su padre era el comandante, usualmente con el grado de capitán ${ }^{5}$.

Pasaba lo mismo en América Latina, muy en particular en la Real Audiencia de Quito. En las compañías fijas hubo un total de ocho cadetes cuyas hojas de vida reposan en el Archivo de Indias; tal es el caso del coronel Juan de Salinas, prócer de la independencia de Quito (1809), quien pasó catorce años con el grado de cadeté .

Para los ejércitos latinoamericanos, el referente histórico militar tiene origen europeo. Se conoce que en el siglo xviı se crearon las escuelas militares, siendo Francia el Estado con mayor trascendencia en este tema, de hecho la palabra cadete proviene del francés cadet, que significa menor o hermano menor.

El historiador colombiano Carlos Arturo Reina nos aclara el panorama de los niños combatientes. Un caso corresponde - en sentido épico - al héroe niño Pedro Pascasio Martínez, un niño de doce años que se enroló al Batallón Rifles, en tiempos de las guerras por la independencia; y el otro caso con la famosa guerra de los Mil Días (1899-1902), cuando se enfrentaron liberales y conservadores. Basta con ver la fotografía publicada en la revista L'Illustration de París, que da cuenta de la presencia en esa guerra civil de niños entre 8 y 11 años, "aproximadamente". Allí se puede comprobar "la emergencia de los niños y los jóvenes en los conflictos colombianos" y su participación en las diversas guerras civiles ${ }^{7}$.

En este contexto de juventudes militares, Mayra Fernanda Rey reconoce que, a pesar de la intervención de misiones militares tanto de Estados Unidos como de Francia, los intentos por establecer una escuela militar en Colombia desde fines del siglo xIx fueron fallidos. El panorama cambió a partir de 1907, con la misión militar chilena, tal como ya lo había hecho en el Ecuador en 1900; en Bogotá se reorganizó la Escuela Militar con jóvenes cuyas edades oscilaban entre los 16 y los 20 años de edad ${ }^{8}$.

4 Historia Universal Santillana, Antigüedad III: Grecia y Roma, t. 4 (Quito: Santillana Ecuador, 2007), 26.

5 Juan Marchena F., Ejército y milicias en el mundo colonial americano (Madrid: Fundación Mapfre, 1992), 31-37. Ver también: María Cristina Vera de Flachs "Notas para la Historia de la Universidad en Argentina" en Revista Historia de la Educación Latinoamericana, n.o. $8,(2006): 65-112$

6 "Informe del capitán Citeli, comandante", Archivo General de Indias, AGI, Quito, legajo 574, folio 554, diciembre de 1797.

7 Carlos Arturo Reina, Jóvenes, reclutas y desertores: la juventud utilizada en Colombia: siglo XIX y XX (1819-1960) (Bogotá: Universidad Distrital Francisco José de Caldas, 2017), 65-68.

8 Mayra Fernanda Rey Esteban, “La educación militar en Colombia entre 1886 y 1907”, Repositorio académico de la Universidad de 
Para el siglo xx, específicamente en el período comprendido entre la primera y segunda guerras mundiales, surgieron movimientos infanto-juveniles en el viejo continente que lograron reclutar miles y hasta millones de adolescentes movidos por el sentimiento fascista y nacionalista: los Fasci Giovanili di Combattimento en Italia; el Frente de Juventudes ("Flechas" y "Pelayos"), grupos de niños falangistas en la España de Franco, cuyas actividades se mezclaban entre lo premilitar, político y religioso; y, la Deutsches Jungvolk (juventud alemana) de Hitler - llamados también los "niños-soldado", cuyas edades oscilaban entre los diez y los catorce años - encargados, al final de la Segunda Guerra Mundial, de remover los escombros de sus ciudades destruidas?.

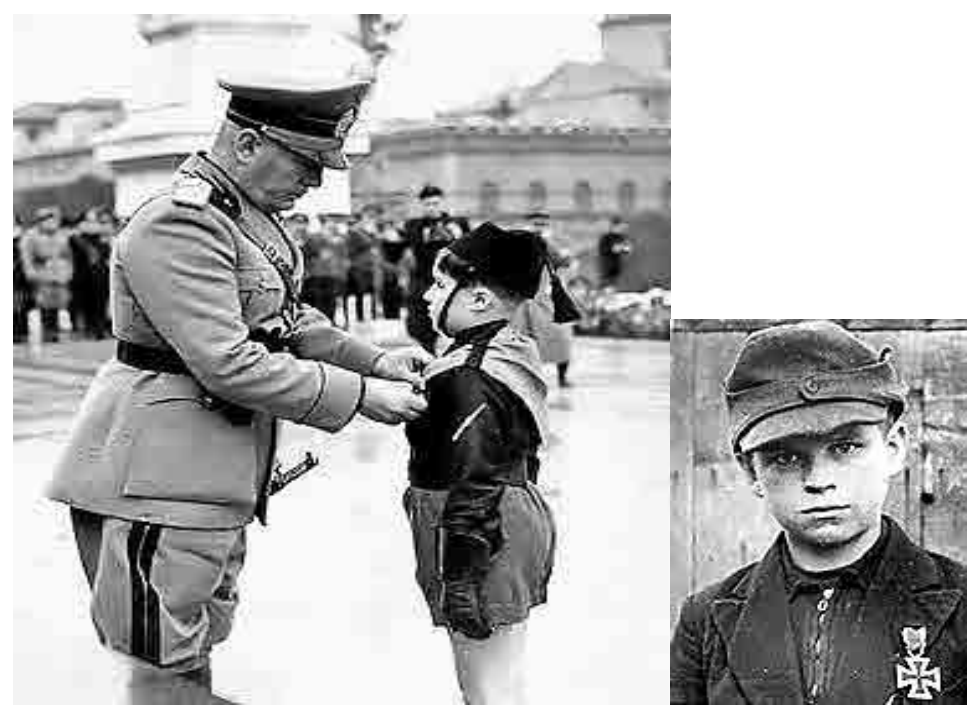

Los niños-soldado de Italia y Alemania. La Primera fotografía es tomada del artículo "Benito Mussolini, el niño mudo y violento que se convirtió en un histriónico tirano y asesino", virilofm.com.ar. En la otra fotografía está el niño de 12 años Alfred Czech, de las juventudes nazis. Foto de David Crary, Buro Laux/Pressens Bild vía AP

En el caso del Ecuador, la historia del Colegio Militar Eloy Alfaro tiene el año de 1902 como fecha de inicio de su funcionamiento como formador de oficiales del Ejército, bajo el mando de la primera misión militar chilena.

En cuanto a nuestro tema central, los testimonios de los oficiales, a continuación, irán marcando el camino:

Pese a estar aceptado en un colegio de curas, en agosto de 1969, el niño Carlos Prócel y su familia pasaron por casualidad frente al Colegio Militar. Leyeron el aviso de apertura a primer curso de educación secundaria, compraron el pros-

Chile (2008), acceso el 5 de octubre de 2018 http://repositorio.uchile.cl/handle/2250/122805.

9 Jacinto Antón, "La factoría de los cachorros nazis", El País Semanal (30 de octubre de 2016) https://elpais.com/elpais/2016/10/30/ eps/1477778806_147777.html. 
pecto, y tras participar en las pruebas físicas, psicológicas, médicas y académicas, Carlos fue aprobado ${ }^{10}$.

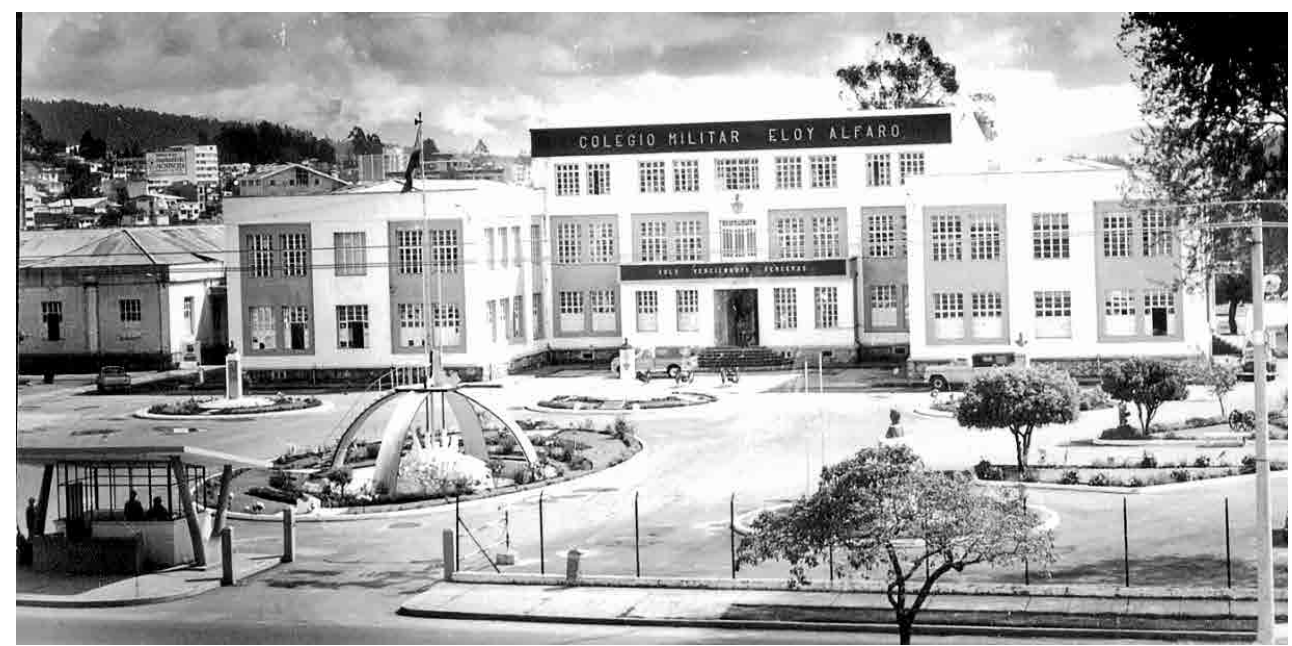

Colegio Militar Eloy Alfaro en 1969. Fuente: Colección fotográfica KAB. El caso de los hermanos Romero fue similar. Su padre, el teniente coronel Jorge Romero, pionero de la Aviación del Ejército ecuatoriano, exhortó a sus hijos Diego y Pablo a que hicieran las pruebas para ingresar al colegio militar, pues había que mantener la dinastía militar en la familia, lo que los niños aceptaron complacidos. Sin embargo, también hubo casos de padres de familia que obligaron a sus hijos a presentarse, pensando en que un colegio militar era una "correccional" donde podrían delegar su rol de padres ${ }^{11}$.

El ingreso de cincuenta y cinco niños a primer curso del Colegio Militar se llevó a cabo en octubre de 1969, luego de haber sido aprobados de acuerdo al listado. Apenas con sus doce años de edad en promedio eran sometidos a un horario de semi internado, esto es, una jornada diaria desde las 6:45 hasta las 17:30, tomando en cuenta que el sábado era dedicado a la instrucción militar: marchas, preparación para desfiles, gimnasias masivas, instrucción formal, saludos y cortesía militar ${ }^{12}$.

10 Entrevista del 15 de agosto de 2017 con el coronel Carlos Prócel, protagonista.

11 Entrevista del $1 .^{\circ}$ de septiembre de 2017 con el coronel Diego Romero, protagonista.

12 Entrevista del 2 de agosto de 2017 con el coronel Juan Reinoso, protagonista. 


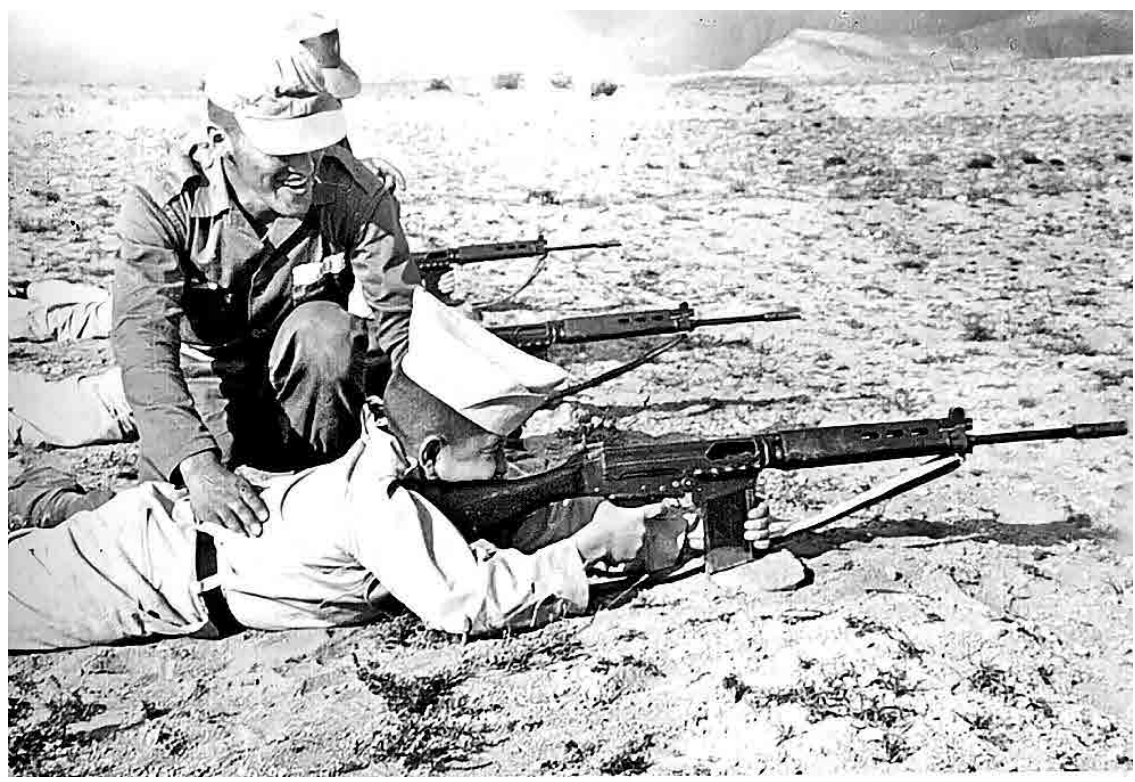

Cadetes del primer curso reciben lecciones de tiro con el fusil Fal, 1970.

Fuente: Archivo fotográfico del Colegio Militar. Quito, Ecuador.

Pasado el primer trimestre (enero de 1970), se publicó el listado de sus notas y antigüedades, lo cual definía el pago de las pensiones. El primer tercio del curso era premiado con la beca completa, el segundo tercio con la media beca y el último tercio pagaba la pensión completa.

Aparte de los cadetes semi internos también había un grupo de cadetes de provincia el internado para los cadetes de provincia cuyos padres obligaban su estancia con carácter de internos. Como suele suceder, lo que para unos era simplemente un castigo, para otros el estar internado era su orgullo, su fortaleza: sufrir el régimen de diana (hora de levantarse) con el toque de trompeta a las cinco de la mañana y el baño de agua fría, hacer la limpieza de sus dormitorios, cumplir con castigos nocturnos... Todo moldeaba su espíritu militar. Así lo sentían. 


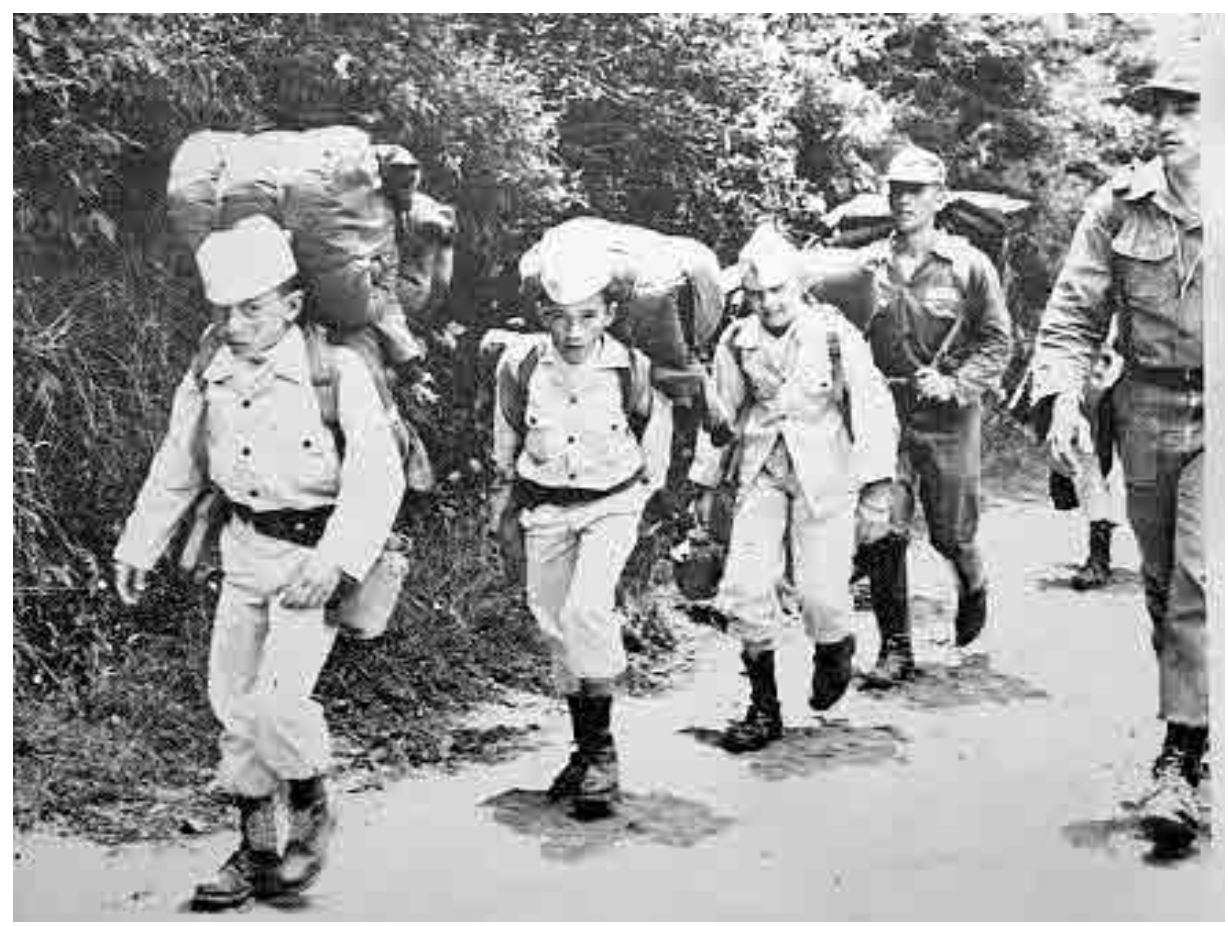

Marcha final, 1972. Fuente: Archivo fotográfico KAB.

Pero para estos niños-cadetes el internado no solo tenía un cuadro de disciplina prusiana. La noche también daba tiempo para emprender algunas travesuras, como ocultar una manguera de suero de hospital entre la olla de la leche de la cocina para poder tomársela sin restricciones; o instalar una línea telefónica en el subsuelo del edificio central para llamar a sus casas a medianoche, con su respectiva tarifa para quienes no eran del grupo de "traviesitos"; o disfrazarse de fantasma para hacer cagar del susto a sus compañeros dando vida a la leyenda del "Cadete sin cabeza"...13.

Un día se produjo un incendio que hizo tocar zafarrancho a todo el colegio. Fue en el subsuelo de uno de los dormitorios, donde se ubicaban los canceles de los cadetes semi internos. Un conocido - y temido- grupo de cadetes caracterizados por sus fechorías y por ser dos o tres años mayores que el resto de niños-cadetes tenía su guarida detrás de los canceles, un espacio equipado con colchones y muy bien adecuado para fumar y dormir. Una tarde se escaparon a su guarida a fumar y se quedaron dormidos. Los colchones y otros enseres quedaron reducidos a cenizas. En el parte del oficial de semana constaba que no se habían presentado desgracias personales. Y claro, uno de estos traviesos era hijo de un oficial de muy alto rango ${ }^{14}$.

13 Entrevista del 4 de septiembre de 2017 con el teniente coronel Gino Rodríguez, protagonista.

14 Entrevista del 2 de agosto de 2017 con el coronel Juan Reinoso, protagonista. 
Eran tiempos en los que la disciplina militar era manejada por dos ejes de aplicación: el Reglamento de Disciplina Militar y los castigos físicos, dando a entender que estos "forjan el carácter" y "despiertan la voluntad del educando"15.

Según el Reglamento, una falta leve equivalía a quince minutos de trote al final del día, y para faltas mayores con la presentación los días domingos, desde medio domingo hasta dos domingos. Sin embargo, lo más usual de la época, eran los castigos derivados del maltrato... Una o varias vueltas a la pista atlética y muchas veces sufrir atropellos por parte de los cadetes de la Escuela Militar. Que se rompan los palos de escoba en las nalgas era algo cotidiano.

El castigo más temido era el famoso "trípode", una donde el cuerpo del cadete se sostenía de sus pies y la cabeza, llevando esta el peso mayor. Y si al trípode se sumaba uno o varios palazos en la nalga, el castigo era completo... y también cotidiano $^{16}$.

En las jornadas académicas los cadetes debían cumplir con un programa en el cual se estudiaban las materias propias de esos niveles, en concordancia con el Ministerio de Educación, Cultura y Deportes de la época: castellano, matemáticas, ciencias naturales, mecanografía, educación para la salud, historia, cívica, dibujo técnico, actividades prácticas e idiomas a elegir: inglés, francés o alemán. Los profesores eran en su mayoría oficiales del Ejército y los profesores civiles tenían el grado de capitán asimilado. Todos los entrevistados recuerdan al doctor Espín, "mi capitán Espín", como el mejor profesor y el más estricto.

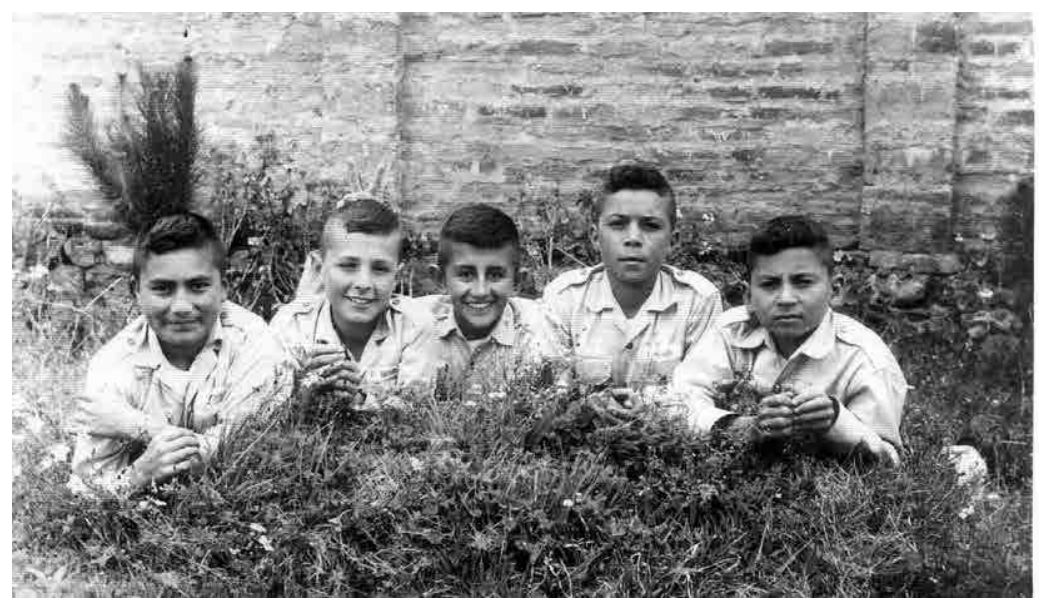

Con el uniforme de clases, 1972. Fuente: Archivo fotográfico KAB.

Este ambiente de formación tenía un desequilibrio hacia lo militar en detrimento de lo académico. Si se compara con un colegio civil, donde el desarrollo de la independencia o la libertad con límites era un legado del Método Montessori, aquí el caso era diferente. El hecho de que haya existido demasiado con-

15 Sandra Milena Fajardo M., "La emergencia de la pedagogía militar en Colombia a mediados del siglo XX: apuntes para su comprensión”, Praxis \& Saber, 8, n. ${ }^{\circ}$ 17, acceso el 5 de octubre de 2018. https://doi.org/10.19053/22160159.v8.n17.2018.4713

16 Entrevista del 2 de septiembre de 2017 con el coronel Jaime Rodríguez, protagonista. 
trol militar en la formación de un niño, próximo a ser adolescente, dejaba en entredicho su relación de lo lúdico con el contexto educativo, haciendo de dicho método pedagógico una utopía lanzada al basurero ${ }^{17}$.

El control disciplinario era ejercido de forma directa por dos brigadieres del último año de la Escuela Militar; aparte de estar encargados de impartir la instrucción militar y la instrucción de desfile, "los Chiquitos" formaban la Quinta Compañía como parte de toda la columna de la Escuela Militar. Incluso viajaban a desfilar en otras ciudades de acuerdo con el calendario cívico ecuatoriano: 9 de octubre en Guayaquil y 3 de noviembre en Cuenca ${ }^{18}$.

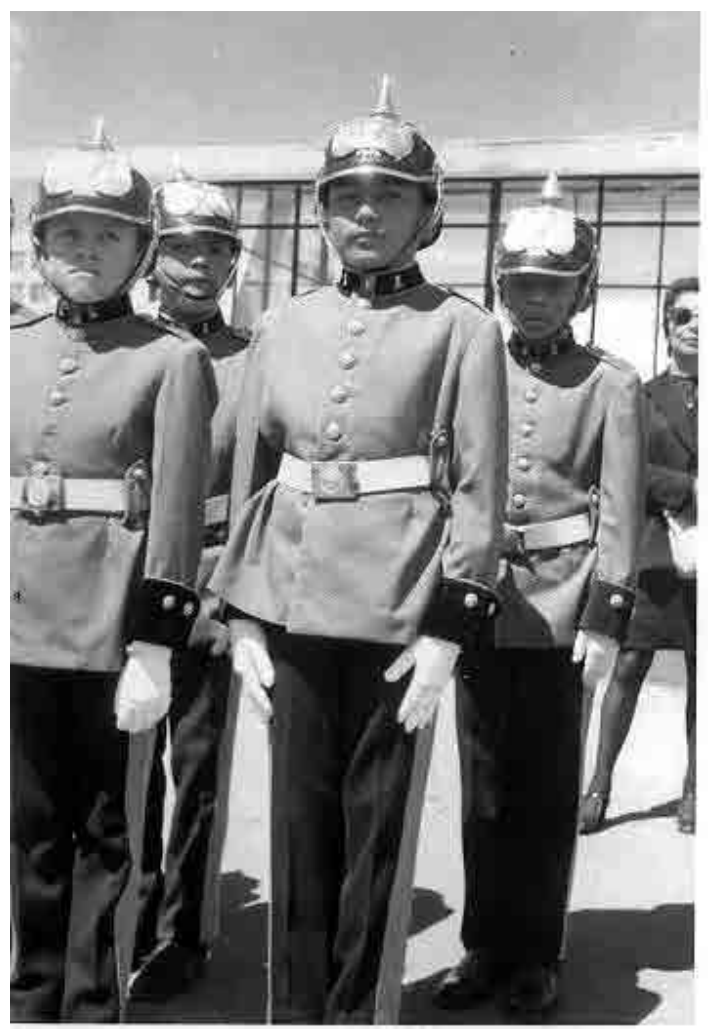

Funte: Con el uniforme de desfile modelo prusiano, 1972. Fuente: Archivo fotográfico KAB.

17 Colaboradores de Wikipedia, s. v. "Método Montessori", Wikipedia, La enciclopedia libre, https://es.wikipedia.org/w/index.php?title=M\%C3\%A9todo_Montessori\&oldid=111316476

18 Entrevista del 19 de julio de 2017 con el general Luis Burbano, brigadier de la Quinta Compañía en 1969. 


\section{CONCLUSIÓN}

La historia de los niños espartanos ha tenido su eco en la historia militar del siglo XX. Está muy claro ver cómo se multiplicaron los grupos de niños involucrados en la formación militar, siendo la juventud alemana el caso más sonado por su ciega veneración a su líder y al fascismo. Se ve que esto no se ha replicado en el Ecuador. Pese a que estos niños - cadetes fueron el globo de ensayo en el fomento del civismo y nacionalismo, no reflejaron en su formación el odio o el resentimiento. Su formación era integral, basada en las esferas física, académica y cívica. A lo mucho odiaban a sus coetáneos civiles. Cosa de chicos. Rasgo de su tiempo.

Cerca del $25 \%$ de este grupo de niños - cadetes fueron hijos de oficiales del Ejército. Esto marca la idea de que su sueño era llegar a ser lo mismo que sus padres. La realidad fue otra. De los 55, apenas tres llegaron al grado de coronel, luego de 30 años de carrera militar. Ninguno alcanzó el grado de general, lo que revela el alto nivel de deserción, especialmente en los cinco años de formación en la Escuela Militar para alcanzar el grado de subteniente.

Dentro de un análisis de aquellos tiempos, estas memorias de vida militar preadolescente permiten revelar lo duro y dogmático que era para ellos estar en un colegio de formación castrense y ser parte analógica de una historia similar a lo que describe Mario Vargas Llosa en su libro La Ciudad y los Perros. Tener que adaptarse a un régimen de dureza con apenas doce años, se convirtió para ellos en un desafío, un sueño, una esperanza. De ahí el lema del Colegio Militar Eloy Alfaro: "Solo venciéndote vencerás".

Los entrevistados recordaban -en primera instancia- los castigos recibidos. Para ellos, el tema de los castigos físicos y corporales era un asunto cotidiano que; en cierto sentido, "no forjó el carácter", sino que permitió cumplir con las obligaciones únicamente por el miedo al castigo, sin que por ello se haya logrado despertar la voluntad hacia el buen comportamiento. Pues claramente las entrevistas reflejaban una suerte de "masoquismo" cuando eran apaleados o colocados en trípode. O sea, el castigo corporal ya se hizo carne, costumbre, hábito en el día a día.

\section{FUENTES}

Archivo fotográfico del Colegio Militar, Quito - Ecuador

Archivo General de Indias, AGI, Sevilla, España, legajo 574, folio 554

Entrevistas

Gral. Luis Burbano, brigadier de la Quinta Compañía, Quito, 25 de junio de 2017

Tcrn. Jorge Romero, padre de familia, Quito, 21 de mayo de 2017

Crnl. Juan Reinoso, protagonista, Quito, 5, 15 de junio de 2017

Crnl. Carlos Teirón Prócel, protagonista, Quito, 30 de junio de 2017

Crnl. Diego Romero, protagonista, Quito, 30 de mayo de 2017

Crnl. Jaime Rodríguez, protagonista, Quito, 20 de junio de 2017

Tcrn. Gino Rodríguez, protagonista, Quito, 10 de junio de 2017 


\section{REFERENCIAS}

Antón, Jacinto. "La factoría de los cachorros nazis". El País Semanal (30 de octubre de 2016) https:// elpais.com/elpais/2016/10/30/eps/1477778806_147777.html.

Fajardo, Sandra Milena. "La emergencia de la pedagogía militar en Colombia a mediados del siglo XX: apuntes para su comprensión". Praxis E Saber, acceso el 5 de octubre de 2018. https:// doi.org/10.19053/22160159.v8.n17.2018.4713.

"Informe del capitán Citeli, comandante". Archivo General de Indias, AGI, Quito, legajo 574, folio 554, diciembre de 1797.

Marchena, Juan. Ejército y milicias en el mundo colonial americano. Madrid: Fundación Mapfre, 1992.

Reina, Carlos Arturo. Jóvenes, reclutas y desertores: la juventud utilizada en Colombia: siglo xIx y xx (1819-1960). Bogotá: Universidad Distrital Francisco José de Caldas, 2017.

Rey, Mayra Fernanda, (2007). "La educación militar en Colombia entre 1886 y 1907", (2008), acceso el 5 de octubre de 2018. http://repositorio.uchile.cl/handle/2250/122805.

Colección Historia Universal. Antigüedad III: Grecia y Roma, t. 4. Quito: Santillana Ecuador, 2007.

Vera de Flachs, María Cristina "Notas para la Historia de la Universidad en Argentina" en Revista Historia de la Educación Latinoamericana, n.o. 8, (2006):65-112

Como citar:

Antonio Bravo, Kléver; Gutiérrez Álvarez, Carlos. “Niñez y Ejército. Historia de los niños-cadetes del Colegio Militar Eloy Alfaro de Quito, Ecuador, 1969 - 1972" Revista Historia de la Educación Latinoamericana. Vol. 20 No. 31 (2018): 71-82

DOI: $10.19053 / 01227238.8582$ 